\title{
Dinâmica do componente arbóreo em função da distância da borda em uma floresta com araucária no Planalto Sul Catarinense, Brasil
}

\author{
Tree component dynamics in function of edge distance in an araucaria forest in \\ "Planalto Sul Catarinense" region, Brazil
}

\author{
Aline Gross ${ }^{\mathrm{I}}$, Ana Carolina da Silva ${ }^{\mathrm{II}}$, Carla Luciane Lima ${ }^{\mathrm{I}}$, \\ Daiany Augusta Paes Martins ${ }^{\mathrm{I}}$, Rogério Nappi Corrêa ${ }^{\mathrm{III}}$, Karine Souza ${ }^{\mathrm{I}}$, \\ Amanda da Silva Nunes ${ }^{\mathrm{I}}$, Aline Pereira Cruz ${ }^{\mathrm{IV}}$, \\ Mariele Alves Ferrer da Silva ${ }^{\mathrm{I}}$, Pedro Higuchi' ${ }^{\mathrm{I}}$
}

\begin{abstract}
Resumo
O presente estudo objetivou analisar a dinâmica do componente arbóreo em floresta com araucária em função da distância da borda, em Santa Catarina, Brasil. Foram realizados levantamentos da vegetação em 2011 e 2015, pelo método de parcelas permanentes, em cinco transeções contendo dez parcelas de $200 \mathrm{~m}^{2}$ cada, sendo as cinco primeiras parcelas $(0-50 \mathrm{~m})$ definidas como setor borda e as cinco últimas (50-100 m) como interior. Em 2011, os indivíduos arbóreos foram inventariados e em 2015 os sobreviventes e mortos foram quantificados e os recrutas incorporados ao levantamento. Foram calculadas as taxas de mortalidade e recrutamento e as espécies foram classificadas em guildas de regeneração. Os resultados demonstraram que a dinâmica da comunidade não diferiu entre os setores, porém as guildas de regeneração apresentaram diferentes performances em função da distância da borda, com espécies existentes em luz favorecidas na borda e espécies tolerantes ao sombreamento no interior. Conclui-se que a fragmentação florestal tem o potencial de impactar negativamente o componente arbóreo na floresta com araucária.
\end{abstract}

Palavras-chave: Efeitos de borda; Floresta Ombrófila Mista; Guildas de regeneração

\begin{abstract}
This study aimed to analyze the tree component dynamics in an araucaria forest in function of fragment edge distance in the Santa Catarina state, Brazil. To do so, we conducted the vegetation surveys in 2011 and 2015, through permanent plots, in five sampling transects, containing ten $200 \mathrm{~m}^{2}$ plots for each one. The first five plots $(0-50 \mathrm{~m})$ were defined as edge sector and the last five plots $(50-100 \mathrm{~m})$ were considered as interior sector. In 2011, tree individuals were surveyed, with the recruit and dead individuals counted in 2015. The rates of mortality and recruitment were determined, and the species were classified into regeneration guilds. The study showed that there was no difference in tree community dynamics between the analyzed sectors, but the regeneration guilds presented different performance in function of edge distance, with light-demanding species succeeding in edge and shade-tolerant species performing better in the interior. We conclude that forest fragmentation has the potential to impact negatively the tree component in Araucaria forest.
\end{abstract}

Keywords: Edge effect; Araucaria forest; Regeneration guilds

\footnotetext{
Engenheira Florestal, Departamento de Engenharia Florestal, Universidade do Estado de Santa Catarina, Av. Luiz de Camões, 2090, CEP 88520000, Lages (SC), Brasil. alinegros@hotmail.com (ORCID: 0000-0003-2496-8852) / carla_engflorestal@yahoo.com.br (ORCID: 0000-0003-2496-8852) / (ORCID: 0000-0002-7943-3962) / dapmflorestal@gmail.com (ORCID: 0000-0001-5186-8107) / karisouza@hotmail.com (ORCID: 0000-0002-03701502) / amandaeng.f@gmail.com (ORCID: 0000-0001-6669-3936) / mariele.ferrer@hotmail.com (ORCID: 0000-0003-2599-7356)

II Engenheiro(a) Florestal, Dr(a)., Professor(a) do Departamento de Engenharia Florestal, Universidade do Estado de Santa Catarina, Av. Luiz de Camões, 2090, CEP 88520-000, Lages (SC), Brasil. carol_sil4@yahoo.com.br (ORCID: 0000-0002-1285-640X) / higuchip@gmail.com (ORCID: 00000002-3855-555X)

III Engenheiro de Aquicultura, Me., Instituto Geral de Perícias, 6ª Mesorregião de Perícias, Rua Alcinda Passos Varela, s/n, Universitário, CEP 88511040 - Lages (SC), Brasil. rogerionc@hotmail.com (ORCID: 0000-0002-1943-0479)

IV Bióloga, Departamento de Engenharia Florestal, Universidade do Estado de Santa Catarina, Av. Luiz de Camões, 2090, CEP 88520-000, Lages (SC), Brasil. a.line_cruz@yahoo.com.br (ORCID: 0000-0002-9279-7293)
} 


\section{Introdução}

O histórico processo de degradação tem imprimido marcas evidentes sobre a biodiversidade e os processos ecológicos em áreas de Floresta Atlântica brasileira (TABARELLI et al., 2010). Dentre os fatores impactantes, merece destaque a fragmentação florestal, de forma que, atualmente, estima-se que restam apenas $26 \%$ de florestas nativas neste que é um importante hotspot de conservação da biodiversidade mundial (REZENDE et al., 2018). Além das consequências deletérias do isolamento, como aquelas relacionadas aos processos reprodutivos (BORGES et al., 2020), outro fator relevante é o efeito de borda, que se expressa a partir das mudanças abióticas e bióticas associadas à distância da interface entre as matrizes florestais e não-florestais, como, por exemplo, as alterações microclimáticas e da composição taxonômica das comunidades biológicas (HENTZ et al., 2018).

A Floresta Ombrófila Mista (FOM) é uma das principais tipologias vegetais da Floresta Atlântica na região sul do Brasil (IBGE, 2012), onde este histórico de degradação é bastante evidente. Em Santa Catarina, a FOM foi intensamente explorada especialmente durante as décadas de 50 e 60 durante o "Ciclo da Araucária". Além disso, devido ao processo de ocupação de terras, houve a conversão da vegetação natural em áreas para atividades agrícolas e pecuárias, industriais, urbanização e, mais recentemente, a silvicultura. Como resultado, houve grande fragmentação de habitat, restando remanescentes florestais com diferentes áreas, formas e estágios de degradação (RIBEIRO et al., 2009), provavelmente afetando a diversidade genética, com prejuízos ecológicos e econômicos.

Diante desse cenário de degradação, estudos sobre a dinâmica de comunidades arbóreas nos remanescentes de FOM são fundamentais, pois permitem avaliar as flutuações na mortalidade e recrutamento que ocorrem em um determinado intervalo de tempo, além de demonstrar a influência das bordas de fragmentos que, geralmente, possuem composição de espécies e estrutura distinta do interior da floresta (GROSS et al., 2018). Porém, ressalta-se que a similaridade ou não entre os setores borda e interior de um fragmento florestal pode estar relacionada com a interferência sofrida ou com o estágio de sucessão desse fragmento. Por exemplo, perturbações crônicas podem funcionar como filtros, promovendo maior homogeneização da vegetação (LÔBO et al., 2011), o que resultaria em menor dissimilaridade entre áreas de interior e bordas.

Dessa forma, estudos dessa natureza são relevantes, pois permitem gerar subsídios para o manejo florestal visando a conservação desses remanescentes florestais. Considerando o contexto apresentado, este estudo teve como objetivo analisar a dinâmica do componente arbóreo em um fragmento de Floresta Ombrófila Mista em Santa Catarina, com o propósito de verificar se existem variações na dinâmica em função da distância da borda.

\section{Material e métodos}

O estudo foi realizado em um fragmento florestal no município de São José do Cerrito, SC, Sul do Brasil. As coordenadas geográficas centrais do fragmento são de $27^{\circ} 44^{\prime} 16^{\prime \prime S}$ e $50^{\circ} 28^{\prime} 51^{\prime \prime O}$, e a altitude é em torno de $900 \mathrm{~m}$ (Figura 1). O clima da região é Cfb, de acordo com a classificação de Köppen, e a temperatura média do mês mais quente não chega a $22^{\circ} \mathrm{C}$ (EMBRAPA, 2016). A vegetação florestal pode ser classificada como Floresta Ombrófila Mista Montana (IBGE, 2012).

A área apresenta histórico de perturbação, com cortes seletivos no passado, até meados da década de 60 , e atividades pecuárias no entorno. A matriz de entorno é predominantemente campestre. Atualmente, o fragmento encontra-se parcialmente protegido por meio de cercas para impedir a entrada de gado.

No ano de 2011, foi realizado o primeiro levantamento no fragmento por Pscheidt et al. (2018). O fragmento foi amostrado pelo método de parcelas permanentes, totalizando 1 ha de área amostrada. Para isso, os autores estabeleceram cinco transeções perpendiculares à borda do fragmento, distanciadas, pelo menos, $100 \mathrm{~m}$ entre si, com a extensão de $100 \mathrm{~m}$ para o interior da 
floresta e a largura de $20 \mathrm{~m}$. Cada transeção foi subdividida em dez parcelas de $10 \mathrm{~m}$ por $20 \mathrm{~m}$ de largura, totalizando 50 parcelas. Foi considerado como setor borda as cinco primeiras parcelas $(0-50 \mathrm{~m})$ de cada transeção e como setor interior as cinco últimas (50-100 m). Dentro das parcelas, foram identificados, marcados e mensurados (CAP - circunferência à altura do peito, medida a $1,30 \mathrm{~m}$ do solo) todos os indivíduos arbóreos com CAP igual ou superior a 15,7 cm.

Figura 1 - Localização geográfica e distribuição das parcelas estudadas ao longo de um gradiente borda-interior de um fragmento de floresta com araucária em Santa Catarina, Brasil

Figure 1 - Geographic localization and distribution of study plots along an edge-interior gradient in an Araucaria Forest fragment in Santa Catarina state, Brazil

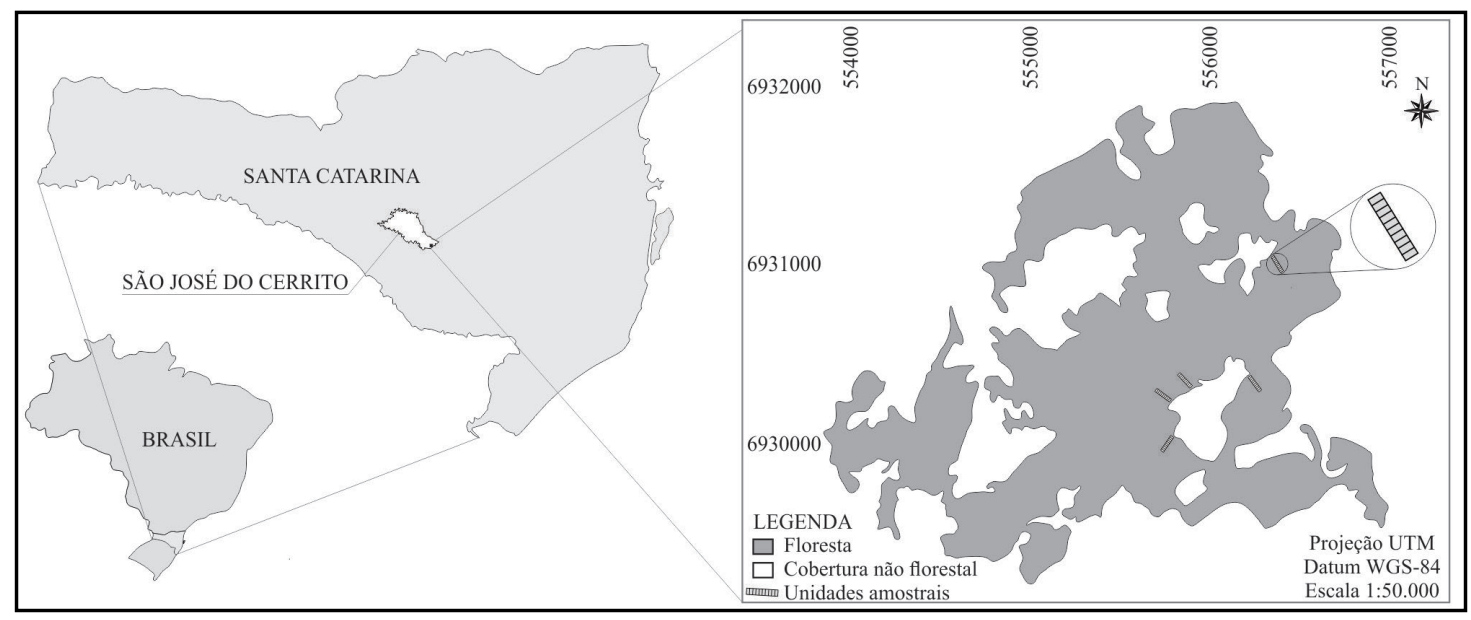

Fonte: Autores (2020)

Para o estudo de dinâmica florestal, quatro anos após o primeiro levantamento (2015), essas 50 parcelas foram reinventariadas. Os indivíduos mortos foram registrados e os sobreviventes, mensurados novamente. Os novos indivíduos que atingiram o nível de inclusão de $15,7 \mathrm{~cm}$ de CAP foram incorporados ao inventário, seguindo a mesma metodologia anterior, e considerados recrutas. As identificações foram realizadas por meio de pareceres de especialistas e literatura especializada. Foram calculadas, em nível de comunidade e de população, para os setores borda e interior, as taxas de mortalidade e recrutamento conforme metodologia utilizada por Gross et al. (2018).

Para verificar a composição das guildas de regeneração das populações de cada setor (borda e interior), as espécies foram classificadas em: pioneiras (P), cujos indivíduos necessitam de luz direta para germinar e se estabelecer, clímax exigente de luz (CEL), que precisam de luz quando jovens para atingir o dossel, e clímax tolerante à sombra (CTS), que são as espécies cujas sementes germinam e crescem sob a sombra, em condições de sub-bosque. Essa classificação foi feita a partir da metodologia usada por Morel et al. (2018).

Foram realizados testes de proporção para verificar se os percentuais de indivíduos mortos e recrutados diferiram ente a borda e o interior. Também foram aplicados testes de quiquadrado a tabelas de contingências para verificar se os padrões de dinâmica das populações relacionaram-se com as guildas de regeneração nos setores borda e interior. As análises dos dados foram realizadas utilizando planilha eletrônica e a linguagem de programação estatística R (R CORE TEAM, 2019). 


\section{Resultados e discussão}

Para a comunidade como um todo, os valores percentuais de indivíduos mortos e recrutados não diferiram significativamente entre os setores (mortos $=9,5 \%$ na borda e $8,8 \%$ no interior, $\mathrm{p}=0,71$; recrutados $=5,0 \%$ na borda e $6,1 \%$ no interior, $\mathrm{p}=0,44$ ), indicando que as taxas demográficas na borda e no interior são semelhantes. Esse resultado difere do padrão observado em estudos realizados em outras áreas do domínio Atlântico, que têm demonstrado padrões distintos de mortalidade e recrutamento entre borda e interior (ver MENDES et al., 2016), sugerindo que os efeitos da fragmentação florestal sobre a dinâmica do componente arbóreo em diferentes fitofisionomias não podem ser generalizados. De fato, Orihuela et al. (2015), no Sul do Brasil, argumentaram que a Floresta Ombrófila Mista, quando comparada com a Floresta Ombrófila Densa, apresenta uma flora intrinsicamente mais resiliente à distúrbios antrópicos, pelo fato de possuir naturalmente mais espécies exigentes em luz e adaptadas a distúrbios.

No entanto, apesar da similaridade do padrão de dinâmica para a comunidade entre os setores avaliados, ao se analisar as taxas demográficas por espécies (Tabela 1), algumas diferenças são evidenciadas, denotando que mesmo supostamente apresentando uma flora mais resiliente, podem existir táxons que respondem de forma distinta ao processo de fragmentação. Das dez espécies mais abundantes em ambos os setores no ano de 2011, na borda se destacaram Caseria decandra Jacq., por apresentar a maior taxa de mortalidade $\left(4,0 \% \cdot \mathrm{ano}^{-1}\right)$, e Cinnamodendron dinisii Schwacke, por apresentar a maior taxa de recrutamento $\left(3,9 \%\right.$. ano $\left.{ }^{-1}\right)$. Considerando as diferenças entre as taxas de recrutamento e mortalidade ainda para o setor borda, também se destacaram Inga virescens Benth., por apresentar elevada mortalidade $\left(2,6 \%\right.$.ano $\left.{ }^{-1}\right)$ com nenhum recrutamento, e Araucaria angustifolia (Bertol.) Kuntze, por apresentar elevado recrutamento $\left(2,4 \%\right.$.ano $\left.{ }^{-1}\right)$, com nenhuma mortalidade.

Tabela 1 - Taxas de dinâmica das populações arbóreas no período de 2011-2015, nos setores de borda e interior de um fragmento de floresta com araucária em Santa Catarina

Table 1 - Dynamics rates of tree populations for the 2011-2015 period, in edge and interior sectors, in an Araucaria Forest fragment, in Santa Catarina state

\begin{tabular}{|c|c|c|c|c|c|c|c|c|c|c|}
\hline \multirow{2}{*}{ Espécies } & \multicolumn{5}{|c|}{ Setor borda } & \multicolumn{5}{|c|}{ Setor interior } \\
\hline & $\mathbf{M}$ & $\mathbf{R}$ & $\mathbf{s}$ & TxM & TxR & $\mathbf{M}$ & $\mathbf{R}$ & $\mathbf{S}$ & TxM & TxR \\
\hline Acca sellowiana (O.Berg) Burret & - & - & 2 & 0,0 & 0,0 & - & 1 & 2 & 0,0 & 9,6 \\
\hline $\begin{array}{l}\text { Allophylus edulis (A.St.-Hil. et al.) Hieron. } \\
\text { Ex Niederl. }\end{array}$ & 2 & 3 & 45 & 1,1 & 1,6 & - & 1 & 15 & 0,0 & 1,6 \\
\hline Allophylus guaraniticus (A.St.-Hil.) Radlk. & - & - & 7 & 0,0 & 0,0 & - & - & 6 & 0,0 & 0,0 \\
\hline Annona rugulosa (Schltdl.) H.Rainer & - & 1 & 7 & 0,0 & 3,3 & 3 & 2 & 4 & 13,1 & 9,6 \\
\hline Araucaria angustifolia (Bertol.) Kuntze & - & 3 & 29 & 0,0 & 2,4 & 1 & 2 & 29 & 0,8 & 1,7 \\
\hline Baccharis semiserrata DC. & 1 & 1 & - & 100,0 & 100,0 & - & - & - & - & - \\
\hline Baccharis uncinella DC. & 3 & 1 & 7 & 8,5 & 3,3 & - & - & - & - & - \\
\hline Banara tomentosa Clos & 1 & - & 5 & 4,5 & 0,0 & - & - & 8 & 0,0 & 0,0 \\
\hline Blepharocalyx salicifolius (Kunth) O.Berg & 2 & - & 4 & 9,6 & 0,0 & 1 & - & 2 & 9,6 & 0,0 \\
\hline Butia eriospatha (Mart. exDrude) Becc. & - & - & 1 & 0,0 & 0,0 & - & - & - & - & - \\
\hline Calyptranthes concinna DC. & - & - & 2 & 0,0 & 0,0 & 1 & - & 12 & 2,0 & 0,0 \\
\hline Campomanesia xanthocarpa (Mart.) O.Berg & - & 2 & 12 & 0,0 & 3,8 & - & 1 & 17 & 0,0 & 1,4 \\
\hline
\end{tabular}


Tabela 1 - Continuação ...

Table 1 - Continuation ...

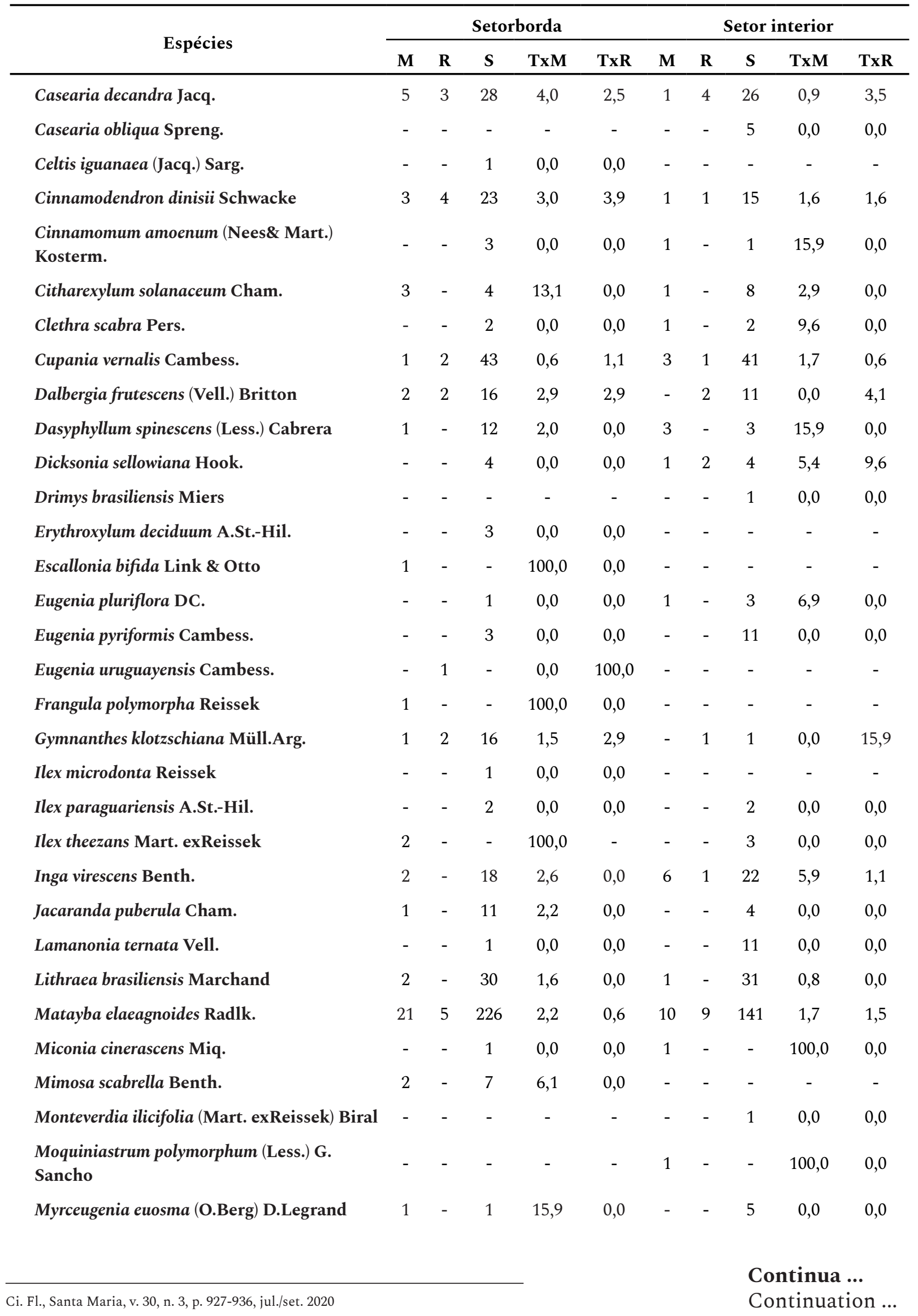


Tabela 1 - Continuação ...

Table 1 - Continuation ...

\begin{tabular}{|c|c|c|c|c|c|c|c|c|c|c|}
\hline \multirow{2}{*}{ Espécies } & \multicolumn{5}{|c|}{ Setorborda } & \multicolumn{5}{|c|}{ Setor interior } \\
\hline & $\mathbf{M}$ & $\mathbf{R}$ & $\mathbf{s}$ & TxM & TxR & $\mathbf{M}$ & $\mathbf{R}$ & $\mathbf{s}$ & TxM & TxR \\
\hline $\begin{array}{l}\text { Myrceugenia glaucescens (Cambess.) } \\
\text { D.Legrand \& Kausel }\end{array}$ & 1 & 1 & 1 & 15,9 & 15,9 & 2 & - & 10 & 4,5 & 0,0 \\
\hline $\begin{array}{l}\text { Myrceugenia oxysepala (Burret) D.Legrand } \\
\text { \& Kausel }\end{array}$ & - & - & - & - & - & - & - & 1 & 0,0 & 0,0 \\
\hline Myrcia hatschbachii D.Legrand & - & 3 & 8 & 0,0 & 7,7 & 2 & 2 & 17 & 2,7 & 2,7 \\
\hline Myrcia selloi (Spreng.) N. Silveira & 1 & - & - & 100,0 & 0,0 & 1 & 1 & 1 & 15,9 & 15,9 \\
\hline Myrcia oblongata DC. & 2 & - & 15 & 3,1 & 0,0 & 1 & - & 9 & 2,6 & 0,0 \\
\hline Myrcia palustris DC. & - & - & 1 & 0,0 & 0,0 & - & - & 3 & 0,0 & 0,0 \\
\hline Myrcia splendens (Sw.) DC. & - & - & 12 & 0,0 & 0,0 & - & - & 7 & 0,0 & 0,0 \\
\hline $\begin{array}{l}\text { Myrcianthes gigantea (D.Legrand) } \\
\text { D.Legrand }\end{array}$ & - & - & - & - & - & - & - & 1 & 0,0 & 0,0 \\
\hline Myrciaria delicatula (DC.) O.Berg & - & 1 & 3 & 0,0 & 6,9 & - & - & 1 & 0,0 & 0,0 \\
\hline Myrrhinium atropurpureum Schott & - & - & 1 & 0,0 & 0,0 & 1 & - & - & 100,0 & 0,0 \\
\hline $\begin{array}{l}\text { Myrsine coriacea }(\mathrm{Sw} \text {.) R.Br. ex Roem.\& S } \\
\text { chult. }\end{array}$ & 3 & - & 5 & 11,1 & 0,0 & 2 & - & 3 & 12,0 & 0,0 \\
\hline Myrsine umbellata Mart. & - & - & 5 & 0,0 & 0,0 & - & 1 & 4 & 0,0 & 5,4 \\
\hline Nectandra lanceolata Nees & - & - & 17 & 0,0 & 0,0 & - & - & 3 & 0,0 & 0,0 \\
\hline Nectandra megapotamica (Spreng.) Mez & 1 & - & 21 & 1,2 & 0,0 & - & 2 & 6 & 0,0 & 6,9 \\
\hline Ocotea puberula (Rich.) Nees & 2 & - & 21 & 2,3 & 0,0 & 1 & - & 10 & 2,4 & 0,0 \\
\hline Ocotea pulchella (Nees \& Mart.) Mez & 2 & - & 17 & 2,7 & 0,0 & 1 & - & 23 & 1,1 & 0,0 \\
\hline Oreopanax fulvus Marchal & - & - & - & - & - & - & - & 1 & 0,0 & 0,0 \\
\hline Picramnia parvifolia Engl. & - & - & - & - & - & - & - & 1 & 0,0 & 0,0 \\
\hline Podocarpus lambertii Klotzsch ex Endl. & - & 1 & 3 & 0,0 & 6,9 & - & 1 & 2 & 0,0 & 9,6 \\
\hline Prunus myrtifolia (L.) Urb. & 1 & 1 & 7 & 3,3 & 3,3 & - & - & 1 & 0,0 & 0,0 \\
\hline Quillaja lancifolia D.Don & - & - & 3 & 0,0 & 0,0 & - & - & 1 & 0,0 & 0,0 \\
\hline Roupala montana Aubl. & - & - & 1 & 0,0 & 0,0 & - & - & - & - & - \\
\hline Rubiaceae sp.1 & - & 1 & - & - & 100,0 & - & - & - & - & - \\
\hline Sapium glandulosum (L.) Morong & 2 & - & 4 & 9,6 & 0,0 & - & - & 1 & 0,0 & 0,0 \\
\hline Schinus sp. & - & - & 1 & 0,0 & 0,0 & - & - & - & - & - \\
\hline Schinus terebinthifolia Raddi & 2 & - & 1 & 24,0 & 0,0 & 1 & - & 2 & 9,6 & 0,0 \\
\hline Scutia buxifolia Reissek & - & - & 1 & 0,0 & 0,0 & - & - & - & - & - \\
\hline Sebastiania brasiliensis Spreng. & - & - & 1 & 0,0 & 0,0 & - & - & - & - & - \\
\hline Sloanea hirsuta (Schott) Planch. Ex Benth. & - & - & - & - & - & - & 2 & 1 & 0,0 & 24,0 \\
\hline Solanum mauritianum Scop. & - & - & - & - & - & - & - & 1 & 0,0 & 0,0 \\
\hline Solanum sanctae-catharinae Dunal & - & - & 5 & 0,0 & 0,0 & 3 & - & 6 & 9,6 & 0,0 \\
\hline
\end{tabular}


Tabela 1 - Conclusão ...

Table 1 - Conclusion ...

\begin{tabular}{lccccccccccc}
\hline & \multicolumn{4}{c}{ Espécies } & \multicolumn{4}{c}{ Setorborda } & \multicolumn{4}{c}{ Setor interior } \\
\cline { 2 - 10 } & M & $\mathbf{R}$ & $\mathbf{S}$ & $\mathbf{T x M}$ & TxR & M & R & S & TxM & TxR \\
\hline Solanum sp. & - & - & 1 & 0,0 & 0,0 & 1 & - & 2 & 9,6 & 0,0 \\
Strychnos brasiliensis Mart. & - & - & 1 & 0,0 & 0,0 & - & 1 & 2 & 0,0 & 9,6 \\
Styrax leprosus Hook. \& Arn. & 1 & 1 & 17 & 1,4 & 1,4 & 1 & 1 & 11 & 2,2 & 2,2 \\
Symphyopappus lymansmithii B. L. Rob. & 1 & - & - & 100,0 & 0,0 & - & - & - & - & - \\
Symplocos uniflora (Pohl) Benth. & 2 & 1 & 2 & 15,9 & 9,6 & - & - & 1 & 0,0 & 0,0 \\
Vernonanthura discolor (Spreng.) H.Rob. & - & - & 3 & 0,0 & 0,0 & 1 & - & 2 & 9,6 & 0,0 \\
Xylosma ciliatifolia (Clos) Eichler & 2 & 1 & 13 & 3,5 & 1,8 & 1 & - & 3 & 6,9 & 0,0 \\
Zanthoxylum kleinii (R.S. Cowan) P.G. & - & 1 & 15 & 0,0 & 1,6 & 1 & - & 16 & 1,5 & 0,0 \\
Waterman & 2 & - & 10 & 4,5 & 0,0 & - & - & 1 & 0,0 & 0,0 \\
Zanthoxylum rhoifolium Lam. & - & - & - & - & - & - & - & 2 & 0,0 & 0,0 \\
NI & 83 & 42 & 794 & 2,5 & 1,3 & 58 & 39 & 603 & 2,3 & 1,6 \\
\hline Total & & & & & & & &
\end{tabular}

Fonte: Autores (2020)

Em que: $\mathrm{M}=$ número de indivíduos mortos, $\mathrm{R}=$ número de recrutas, $\mathrm{S}=$ número de sobreviventes, $\mathrm{Tx} \mathrm{M}=\mathrm{taxa}$ de mortalidade $\left(\%\right.$.ano $\left.{ }^{-1}\right)$, $\mathrm{TxR}=$ taxa de recrutamento $\left(\%\right.$.ano $\left.{ }^{-1}\right)$.

No interior, Inga virescens se destacou por apresentar a maior taxa de mortalidade $5,9 \%$. ano ${ }^{-1}$ ), indicando ser uma espécie com elevada mortalidade em ambos os setores, e Casearia decandra, pela maior taxa de recrutamento $\left(3,5 \% \cdot \mathrm{ano}^{-1}\right)$. Assim, nota-se uma tendência de diferentes padrões de dinâmica para a maioria das espécies, o que pode explicar a existência da divergência florística-estrutural como observada por Pscheidt et al. (2018) para a mesma área. Esse resultado possivelmente está relacionado à existência de diferenças ambientais associadas à distância da borda e às exigências ecológicas das espécies. Por exemplo, diferenças microclimáticas podem explicar o maior incremento populacional de Araucaria angustifolia na parte mais marginal do fragmento, considerando que esta é uma espécie com grande capacidade de estabelecimento em áreas mais abertas (LONGHI et al., 2008), e pode explicar também a maior mortalidade na borda e recrutamento no interior de Casearia decandra, que é uma espécie típica do sub-bosque da FOM (MEYER et al., 2013).

A ideia de que a heterogeneidade na organização florístico-estrutural no fragmento em função do efeito de borda seja o resultado da dinâmica diferencial das espécies é reforçada pelo fato dos padrões de dinâmica observados não terem sido independentes das guildas de regeneração em ambos os setores ( $p<0,1$, Tabelas 2 e 3). Na borda (Tabela 2), o incremento populacional se deu, preferencialmente, a partir de espécies clímax exigentes em luz (CEL = nove espécies observadas, maior que 6,57 esperadas) e o decremento se concentrou, predominantemente, em espécies pioneiras ( $\mathrm{P}=$ nove espécies observadas, maior que 6,75 esperadas). Apesar de se esperar também o aumento de espécies pioneiras na borda, ressalta-se que esse grupo possui a dinâmica bastante rápida, o que explica sua redução nesse setor. Além disso, considerando-se que o processo de fragmentação na área é antigo, é esperado borda mais estável, com espécies CEL substituindo as pioneiras. 
Tabela 2 - Tabela de contingência contendo o número de espécies observado e esperado (entre parênteses), de acordo com o teste de qui-quadrado $\left(X^{2}=8,96, p=0,06\right)$, considerando as guildas de regeneração e as tendências de dinâmica das populações arbóreas amostradas no setor borda de um fragmento de floresta com araucária em Santa Catarina

Table 2 - Contingency table containing the number of observed and expected species (in parentheses), according to the chi-square test $\left(\mathrm{X}^{2}=8.96, p=0.06\right)$, considering the regeneration guilds and dynamic trends of tree populations sampled in the edge sector of an araucaria forest fragment in Santa Catarina

\begin{tabular}{lcccc}
\hline & \multicolumn{3}{c}{ Guildas de Regeneração } & Total \\
\hline Tendências de dinâmica & $\mathbf{P}$ & CEL & CTS & \\
\hline$\uparrow$ & $0(2,75)$ & $9(6,57)$ & $2(1,68)$ & 11 \\
$\leftrightarrow$ & $9(8,50)$ & $17(20,31)$ & $8(5,19)$ & 34 \\
$\downarrow$ & $9(6,75)$ & $17(16,13)$ & $1(4,13)$ & 27 \\
\hline Total & 18 & 43 & 11 & \\
\hline
\end{tabular}

Fonte: Autores (2020)

Em que: $\mathrm{P}$ = pioneira; $\mathrm{CEL}$ = clímax exigente em luz; CTS = clímax tolerante a sombra; $\uparrow$ = incremento, $\leftrightarrow=$ estabilidade e $\downarrow=$ decremento

Tabela 3 - Tabela de contingência contendo o número de espécies observado e esperado (entre parênteses), de acordo com o teste de qui-quadrado $\left(X^{2}=10,86, p=0,02\right)$, considerando as guildas de regeneração e as tendências de dinâmica das populações arbóreas amostradas no setor interior de um fragmento de floresta com araucária em Santa Catarina

Table 3 - Contingency table containing the number of observed and expected species (in parentheses), according to the chi-square test $\left(\mathrm{X}^{2}=10.86, p=0.02\right)$, considering the regeneration guilds and dynamic trends of tree populations sampled in the interior sector of an Araucaria forest fragment in Santa Catarina state

\begin{tabular}{lcccc}
\hline & \multicolumn{3}{c}{ Guildas de Regeneração } & Total \\
\hline Tendências de dinâmica & $\mathbf{P}$ & CEL & CTS & \\
\hline$\uparrow$ & $1(2,33)$ & $8(8,54)$ & $4(2,13)$ & 13 \\
$\leftrightarrow$ & $2(5,19)$ & $22(19,04)$ & $5(4,76)$ & 29 \\
$\downarrow$ & $9(4,48)$ & $14(16,42)$ & $2(4,10)$ & 25 \\
\hline Total & 12 & 34 & 11 & \\
\hline
\end{tabular}

Fonte: Autores (2020)

Em que: $\mathrm{P}=$ pioneira; $\mathrm{CEL}$ = clímax exigente em luz; CTS = clímax tolerante a sombra; $\uparrow$ = incremento, $\leftrightarrow=$ estabilidade e $\downarrow=$ decremento.

Já no interior (Tabela 3), o incremento populacional foi preferencialmente de espécies tolerantes ao sombreamento (CTS = quatro espécies observadas, maior que 2,13 esperadas) e o decremento populacional se deu, preferencialmente, em espécies pioneiras $(\mathrm{P}=$ nove espécies 
observadas maior que 4,48 esperadas). Nesse caso, tanto o aumento de CTS como decremento em P são esperados para esse setor. Dessa forma, o padrão aqui encontrado corrobora com outros já observados para áreas de FOM no Sul do Brasil (SCHAADT; VIBRANS, 2015; HENTZ et al., 2017; SCIPIONI et al., 2018), demonstrando a influência do tamanho da área do remanescente e do efeito de borda sobre a distribuição de guildas de regeneração de espécies arbóreas, o que reforça a ideia dos efeitos negativos da fragmentação sobre o componente arbóreo dessa importante fitofisionomia da Floresta Atlântica.

\section{Conclusões}

Observou-se que no fragmento de FOM estudado, apesar do percentual de indivíduos arbóreos mortos e recrutados não terem diferido entre a borda e o interior, houve o favorecimento de guildas de regeneração natural distintas entre os setores, com espécies exigentes em luz, como Araucaria angustifolia, se destacando na parte marginal do fragmento, e espécies tolerantes ao sombreamento, como Casearia decandra, se destacando no interior. Assim, infere-se que processos de fragmentação florestal podem ter impacto significativo sobre comunidades de espécies arbóreas em áreas de FOM, com o potencial de resultar na homogeneização biológica em áreas com grande proporção de bordas.

\section{Agradecimentos}

À EPAGRI/Lages, por ter fornecido a área de estudo. À FUMDES, pela concessão de bolsa à primeira autora do trabalho. À FAPESC, pelo apoio por meio do PAP/UDESC. Ao CNPq, pela concessão de bolsa de produtividade para o segundo e o último autores e financiamento do trabalho.

\section{Referências}

BORGES, D. B. et al. Changes in fine-scale spatial genetic structure related to protection status in Atlantic Rain Forest fragment. Journal for Nature Conservation, Amsterdam, v. 53, p. 125784, 2020.

EMBRAPA. Clima. [S. l., 2016]. Disponível em: www.cnpf.embrapa.br/pesquisa/efb/clima.htm. Acesso em: 15 abr. 2016.

GROSS, A. et al. Fragmentation as a key driver of tree community dynamics in mixed subtropical evergreen forests in Southern Brazil. Forest Ecology and Management, Amsterdam, v. 411, p. 20-26, mar. 2018.

HENTZ, A. M. K. et al. Edge effect on a tree community in an Araucaria Forest Fragment in Brazil. Floresta, Curitiba, v. 47, n. 4, p. 407-416, 2017.

HENTZ, A. M. K. et al. Edge effect on the spatial distribution of trees in an Araucaria Rainforest fragment in Brazil, Rodriguésia, Rio de Janeiro, v. 69, p. 1937-1952, 2018.

IBGE. Manual Técnico da Vegetação Brasileira. Rio de Janeiro, 2012. 271 p.

LÔBO, D. et al. Forest fragmentation drives Atlantic forest of northeastern Brazil to biotic homogenization. Diversity and Distributions, Hoboken, v. 17, n. 2, p. 287-296, 2011.

LONGHI, S. J. et al. Fatores ecológicos determinantes na ocorrência de Araucaria angustifolia 
e Podocarpus lambertii, na Floresta Ombrófila Mista da FLONA de São Francisco de Paula, RS, Brasil. Ciência Rural, Santa Maria, v. 40, n. 1, p. 57-63, fev. 2010.

MENDES, P. G. A. et al. Dynamics and Edge Effect of an Atlantic Forest Fragment in Brazil. Floresta e Ambiente, Seropédica, v. 23, n. 3, p. 340-349, set. 2016.

MEYER, L. et al. Regeneração natural na Floresta Ombrófila Mista em Santa Catarina. In: VIBRANS, A. C. et al. Inventário Florístico Florestal de Santa Catarina. Floresta Ombrófila Mista. Blumenau: [s. n.], 2013. v. 3. p. 191-222.

MOREL, J. D. et al. Functional characterisation of an anthropized atlantic forest fragment. Journal of Tropical Forest Science, Kepong, v. 30, n. 4, p. 537-545, 2018.

ORIHUELA, R. L. L. et al. Markedly divergent tree assemblage responses to tropical forest loss and fragmentation across a strong seasonality gradient. PlosOne, São Francisco, v. 10, n. 8, p. e0136018, 2015.

PSCHEIDT, F. et al. Efeito de borda como fonte da heterogeneidade do componente arbóreo em uma floresta com araucárias no Sul do Brasil. Ciência Florestal, Santa Maria v. 28, n. 2, p. 601612, jun. 2018.

R CORE TEAM. R: a language and environment for statistical computing. Vienna, [2019]. Disponível em: http://www.r-project.org/. Acesso em: 11 abr. 2019.

REZENDE, C. L.; SCARANO, F. R.; ASSAD, E. D.; JOLY, C. A.; METZGER, J. P.; STRASSBURG, B. B. N.; TABARELLI, M.; FONSECA, G. A.; MITTERMEIER, R. A. From hotspot to hopespot: An opportunity for the Brazilian Atlantic Forest. Perspectives in Ecology and Conservation, Amsterdam, v. 16, p. 208-214, 2018.

RIBEIRO, M. C.; METZGER, J. P.; MARTENSEN, A. C.; The Brazilian Atlantic Forest: How much is left, and how is the remaining forest distributed? Implications for conservation. Biological Conservation, Amsterdam, v. 142, n. 6, p. 1141-1153, 2009.

SCHAADT, S. S.; VIBRANS, A. C. O uso da terra no entorno de fragmentos florestais influencia a sua composição e estrutura. Floresta e Ambiente, Seropédica, v. 22, n. 4, p. 437-445, 2015

SCIPIONI, M. C.; NETO, A. L.; SIMINSKI, A.; SANTOS, V. Forest edge effects on the phytosociological composition of an Araucaria Forest fragment in Southern Brazil. Floresta, Curitiba, v. 48, n. 4, p. 483-492, 2018.

TABARELli, M.; AGUIAR, A. V.; RIBEIRO, M. C.; METZGER, J. P.; PERES, C. A. Prospects for biodiversity conservation in the Atlantic Forest: Lessons from aging human-modified landscapes.

Biological Conservation, Amsterdam, v. 143, p. 2328-2340, 2010. 